\title{
INTRODUCTORY OF BLOCK CHAIN TECHNOLOGY AND APLICATION FOR EDUCATION
}

\author{
Rutheesh Kumar R \\ B.Sc CS-Cloud Technology \\ iNurture Educational Solutions, Bangalore.
}

\author{
Syed Mohamed Mujammil S U \\ B.Sc CS-Cloud Technology \\ iNurture Educational Solutions, Bangalore.
}

\author{
Mohammed Harun Babu R \\ Assistant professor Data Science \& AI \\ iNurture Educational Solutions, Bangalore.
}

\begin{abstract}
Like Artificial Intelligence, Machine learning and Biosystematics there are so many emerging technologies are ruling the world today. One of the major technology were the people are going into was Block Chain. It is the process of securing the information or website of specific individual or institution. This Block Chain Technology mainly deals with Bit Coins. In August 2014, the file size of bit coin block chain, containing records of all transactions that took place on the network, reached 20 GB.

This paper describes the five main features of Block Chain including Decentralized Technology, Enhanced Security, distributed Ledgers etc. A Block Chain is basically a digital transaction ledger, duplicated and distributed on the database across the entire network of computer systems. Finally, this paper deals with the Comparison of Block Chain and Bit coin. After that, the importance and the impact of Bit coin in the sector of Block Chain have been explained.
\end{abstract}

Keywords-Block Chain, Bit coin, Crypto currency, Security, distributed ledgers

\section{INTRODUCTION}

If people around the last fifty years have been following financial services, making investments or digital currency, they may be comfortable with "Block Chain", the record keeping innovations behind the bit coin system. And there is a good possibility that it makes already enough sense. The block chain is not that much tough as its sounds [1]. This is one of the technologies which make people's information more secured in recent years. Block Chain idea first shot to prominence in October 2008, as part of a Bit coin initiative, aimed at creating Peer to Peer money beyond lenders. Bit Coin has provided the strategies to assist to old age human confidence problem. The underlying Blockchain technology enables, us to trust the system's outputs without believing a certain actor inside. Block chain is nothing but the interconnection or the cycle between the blocks. Blocks refer to the information of people or the information in computers and chain refers to the people's database. In simpler words, this Block chain is mainly based on three systems. They are distributed system, decentralized systems and public ledger systems. "Blocks" are composed of virtual sources of evidence on the crypto-currency.

Block Chain is also referred as Distributed Ledger Technology (DLT), by the usage of centralized power and encryption cryptography makes the heritage of every digital asset unchangeable and clear. While explaining about Block Chain, there is one main basic terminology called Miners. Without this component, the possibility of Block chain, cryptocurrency and bit coins would have been very low[2]. Miners use particular technology to solve the immensely complicated math problem of finding a nonce which produces approves password. Because the nonce is only 32 bits and the hash is 256, totally there are about 400 million possible combinations of nonce-hash which need to be mined well before right one is found. If this begins to happen, it is said that miners found the "Glowing nonce" and their block is posted to the ledger.

By this, Blockchain provides a common state surface, a univer sal dataframe that every actor can respect, even if they may no $t$ know each other or trust one another.

The modern method of data deduplication and data manageme nt also eliminates the double spending issue of transfer of existing value over the Internet.It includes every solitary track of all transactions. Crypto currency is an illustration of the BlockChain's most consumers. Blockchain Technology fir st came to platform when in 2008. A person or group named 'Satoshi Nakamoto' published a white paper on 'Bit Coin' which represents a Peer to Peer electronic cash system[3]. In this paper, we are aimed to explain about the various features and components involved in Block Chain.

This paper is divided into the following sections, Section II explains the history of block chain, followed by a brief 


\section{International Journal of Engineering Applied Sciences and Technology, 2020 \\ Vol. 5, Issue 2, ISSN No. 2455-2143, Pages 543-548 \\ Published Online June 2020 in IJEAST (http://www.ijeast.com)}

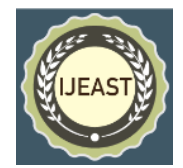

introduction of what is a block chain in section III. Section IV explains the features of block chain technology, advantage and disadvantage has been explained in section $\mathrm{V}$ and finally in section VI the comparative explanation of Bit coin vs. Block chain has been explained

\section{HISTORY OF BLOCKCHAIN}

In 1991 Stuart Haber and 'W.Scott Stornetta' described the first work on a cryptographically secured chain of blocks. They wanted to implement a system which could not manipulate document timestamps. Later in 1992, Merkle Trees were incorporated into the design by Bayer, Haber and Stornetta, which improved its efficiency by allowing several certificates to be collected into one block [4].

In 2008 an individual or group of people known as Satoshi Nakamoto conceptualized the first block chain. "Nakamoto" greatly improved the design using a hash cash like method of time stamping blocks without needing a trusted party to sign them and adding a difficult parameter to control the rate at which blocks are added to the chain. Nakamoto adopted the concept the following year as a central component of the crypto-currency Bit coin, where it acts as the public ledger for all network transactions [5].

In August 2014, the file size of bit coin block chain, containing records of all transactions that took place on the network, reached $20 \mathrm{~GB}$ (gigabytes). The size had grown to almost $30 \mathrm{~GB}$ in January 2015, and the bit coin block chain grew from 50 GB to 100 GB in size from January 2016 to January 2017. As of early 2020, the ledger size had exceeded 200 GB.

In May 2018, Gartner found that only $1 \%$ of CIOs indicated any type of block chain adoption within their organizations, and only $8 \%$ of CIOs were in the short-term "planning or looking at the active block chain experimentation" [6].

\section{BLOCKCHAIN}

Block Chain is a network that stores information in a way that makes it difficult or impossible for the network to modify, hack or cheat. A Block Chain is basically a digital transaction ledger, duplicated and distributed on the database across the entire network of computer systems. Through block in the chain includes a number of transactions, and each time a new transaction takes place on the Block Chain, a record of that transaction is added to the ledger for each participant. Block Chain is a type of DLT in which an immutable cryptographic signature called a hash is used to record transactions.

\section{A. Distributed Ledgers}

Any printed book which holds transaction data is a ledger. These can also look like a ledger. Block Chain is a technology which functions like a ledger. Actually it operates like a distributed ledger, where each Block Chain member owns a copy. A distributed ledger also known as a shared ledger or distributed ledger or DLT is a network of globally dispersed replicated, exchanged, and coordinated digital data through various countries, or institutions. There is no central administrator or centralized data storage. It needs a peer-topeer network, as well as consensus algorithms to ensure node wide replication. The Block Chain framework is one type of distributed ledger architecture which can be either public or private.

The distributed ledger system is spread across multiple nodes (systems) on a peer to peer network, where each replicates and saves an identical ledger copy and updates itself separately. The biggest drawback of this is the absence of centralized control. When a ledger update occurs, the new transaction is created by each node, and then the nodes vote by consensus algorithm on which copy is correct. When there is a consensus, all the other nodes update themselves with the latest, accurate copy of the ledger. The security is accomplished by means of cryptographic keys and signatures [14].

\section{B. Digital Assets}

Block Chain assets are a form of crypto currency or digital asset. Some of them represent stakes in a given project or business. Nevertheless, Block Chain assets are digital, are your sole ownership and are freely transferable to any individual at any time. Digital assets involve almost any digital content: stock listed crypto currency tokens, training materials or audio courses, E-books, various documents, photos, databases, photographs, account top-up card information, access codes, apps, website models, marketing swipe files and so on.

Block Chain Uses Cases in Digital Resources, Coins Tokens and Payments. The ability to represent assets on a decentralized network and conduct transactions using opensource Block Chain technology enables new markets for previously illiquid assets, which decreases post-trade risk which expense [15].

\section{Transaction}

A Block Chain Transaction is a record of transactions in Block Chain. Just like in MySQL database you store a record. Unless they are familiar with the Server, one will know only the basics of Block Chain. It is a base of data. Transactions are stored in the form of blocks, and the blocks form a Blockchain chain. Block Chain transaction is a term referring to a transaction that takes place with the help of a structure Block Chain based. Each operation implies that data or digital currency is transferred between different nodes [16].

\section{Contract}

The recent invention of Block Chain technology revived the notion and encouraged the formation of smart contracts, initially envisaged by SZABO in 1994 (e.g., Tapscott and Tapscott (2016)). Although a widely agreed concept for smart 
contracts has yet to be achieved, their core functionality has clear contracting on contingencies has been reached on the basis of a decentralized consensus, with low cost and more automated implementation.

This leads to a natural functional concept of smart contracts such as Smart contracts are digital contracts that require terms based on decentralized agreement that are deceptive and usually self-enforcing through automated execution [17].

\section{FEATURES OF BLOCKCHAIN}
A. Decentralized Technology
B. Enhanced Security
C. Distributed Ledgers
D. Consensus
E. Faster Settlement

\section{A. Decentralized Technology}

Naturally Block chains are not going to be a cure all the ills of society. There can never be any single instrument or technology. But embracing them on a large societal scale could theoretically combat some of Ito's fears about Silicon Valley's reductionist path of tech evangelism and outsized confidence in artificial intelligence.

First, Silicon Valley's financial monopolization of all the data and value found in the Web 2.0 environment may start redistributing to individual users in Web 3.0.4. Second, Bit coin facilitated social decentralization may theoretically redistribute and re-democratize patterns of human involvement and cooperation. Third, Block Chains are not governed by a central authority, but by the entire network of users, who set the rules for participation themselves and may choose to develop the protocol by consensus and this makes them censorship resistant and potentially more versatile for large numbers of people than most other decision-making mechanisms [7].

The core story of humanity was the difficulty of organizing groups of people and getting them to act in efficient, cooperative ways. A common argument about the potential for Block Chain technology to promote social decentralization is that it might transfer control from centers major metropolises, states, large hierarchical organizations and business to the edges. Decentralization is also a social challenge. Everyone living on Earth today has operated under the system of hierarchy and top-down command and control, and we continue to rely on it as organizational modes.

There are moments when there is a great urge to revert to these familiar forms of communication, and the transition to a less centralized social model of natively digital resources will need to be a deliberate one made several moments over by the network participants. They don't work towards a single vision or goal, an optimization endpoint and a singularity works as much as they're looking for efficient pathways and ways to freely transact [8].

\section{B. Enhanced Security}

While considering one of the most disruptive innovations across industries, Block Chain most definitely has the ability to cut costs, increase accountability, increase protection and, among others, improve performance. The primary advantage of using Block Chain as a platform is improving the data protection it offers. As the name implies, a Block Chain is a chain of digital "blocks" which contain transaction records.

Through block is connected before and after all of the blocks. This makes it impossible to alter a single record because to escape detection, a hacker will need to modify the block containing that record, as well as those connected to it. This alone does not seem like much of dissuasion but Block Chain has several other inherent features that include additional protections. The records are protected through cryptography on a Block Chain. Network participants have their own private keys that are assigned as personal digital signature to the transactions they make and conduct [9].

If a record is modified, the signature is invalid and the peer network should know this immediately. Early warning is crucial in preventing additional damage. Unfortunately, Block Chains are decentralized and spread through peer to peer networks for those creative hackers, which are continually updated and kept in sync. Because they're not in a central location, Block Chains don't have a single failure point and can't be changed from a single computer.

It would take vast quantities of computational power to access and change all instances of a certain Block Chain (or at least a 51 percent majority) at the same time. There was some debate as to whether this means that smaller Block Chain networks could be vulnerable to attack, but a decision was not reached. Anyway, the larger your network is, the more resistant your Block Chain is to abuse.

Block Chains have some attractive features at a glance which will help you protect your transaction data. However, when you choose to use a Block Chain for companies, there are other criteria and specifications to remember [10].

\section{Distributed Ledgers}

Block Chain recently received a lot of attention due mostly to Bit coin and other crypto currencies, but distributed ledgers didn't get the same degree of emphasis. There really seems to be a lot of confusion about the differences between the two. And then we add Bit coin to the mix, and the situation muddles even more. But as we will discuss below, it is actually relatively easy to understand distributed ledger technology.

A distributed ledger is essentially a database that operates over multiple locations or between multiple participants. Most companies use a centralized database which resides at a fixed 
location. But a distributed ledger keeps third parties out of the loop, making them very attractive [11].

\section{Consencus}

A consensus mechanism is a fault tolerant mechanism that is used in computer and Block Chain systems to reach the required agreement among distributed processes or multi agent systems, such as crypto currencies, on a single data value or a single network state. This is also useful in record-keeping.

For any centralized system, such as a database that contains key driving license details for one country, a central administrator has the authority to manage and update the database. The task of making certain changes - such as adding, deleting, updating names of people who applied for those licenses has been carried out by a central authority that retains the sole burden of maintaining legitimate records [12].

\section{E. Faster Settlement}

Block Chain will make settlement of assets plausibly quicker and more versatile. To speed up the settlement process it can bypass existing intermediaries. The platform also helps time sensitive creditors to pay additional transaction fees sooner to complete settlement. First review the basic idea of how to use Block Chain technology to settle the security trades is useful. A securities settlement scheme enables the transfer between investors of legal ownership of the financial properties. This role has historically been performed by a trusted third party. This party maintains a centralized ledger, which documents securities ownership and the transition there Accounts of buyers and sellers after each transaction are credited and debited [13].

Distributed ledger technology (DLT) often simply referred to as "Block Chain" which allows the record of transaction history to be verified, updated, and stored without the use of a designated third party. It is built on a single ledger spread among several different parties, but modified without Usage of third party approved. It relies on a single ledger that is spread by several different parties, but is maintained without a central administrator being dedicated.

There are two baseline DLT models. The first is trustless, where anyone can access the ledger and potentially update it. Consequently it is often referred to as a Block Chain without permission. In the alternative edition many organizations or individuals are entrusted with direct access to and upgrading the Block Chain. Hence, the widely used term is Block Chain with confidence or Block Chain enabled.

\section{ADVANTAGES OF BLOCKCHAIN}

Block Chain technology has the fundamental advantages of decentralization, peer to peer, immutability etc. Some of their major advantages are listed below.

\section{A. Decentralized}

Decentralization is also its main benefit, as one of the most important features of the Block Chain. Instead of being stored in any single location, Block Chain system is totally decentralized, ensuring that an overarching authority cannot advance its own agenda and regulate the network. In addition to being a fair distribution network, decentralization often translates into more stability [18].

\section{B. Peer to Peer network}

The network becomes stronger with growing numbers of participants as information is shared and continuously collected between network participants. A P2P network is part and parcel of Block Chain technology. The term "peer" refers to the Block Chain networked computing system. In this network a user simultaneously makes use of and provides the foundation of the network. Growing peer is called a node, and each is deemed equal.

A peer provides other users with part of resources, such as bandwidth, processing speed and disk capacity, without any servers or hosts requiring central coordination. Even though nodes are similar, they may assume different roles of a full node, or as a miner. In the case of a full node, the entire Block Chain in the linked network will be copied into one single computer. This ensures that Block Chain data cannot be damaged or lost as it requires the destruction of a complete node within the network [19].

\section{Immutability}

Once each block is cryptographically locked, copying, deleting or editing is impossible, ensuring the digital ledger is immutable. Because of the very existence of network decentralization, key points of error or failure within the system can never occur. With the absence of weak points within the hacking program, there could be zero malicious chances, which will improve the security of the network. In addition, each transaction needs digital signatures by both private and public keys that use different cryptographic schemes to ensure maximum encryption.

\section{Open Source}

Block Chain is open source software, the entry barriers are reduced, which contributes to a stronger developer base and increased transparency. Open source software is freely available, created collaboratively, transparently developed and released for the benefit of the world rather than being the property of a single entity or person with the intention of making a profit. No single person or company creates, sells or owns the software in the open source software development process which eliminates any chokepoints or bottlenecks. 


\section{International Journal of Engineering Applied Sciences and Technology, 2020 \\ Vol. 5, Issue 2, ISSN No. 2455-2143, Pages 543-548 \\ Published Online June 2020 in IJEAST (http://www.ijeast.com)}

\section{E. Trust}

As the identity of the user remains secret, the safe network helps us to communicate openly with another. Total transparency and confidentiality of transactions are among the top benefits of the Block Chain technology. Maintaining privacy and anonymity for several reasons is important for the participants. The address is given out with every transaction on centralized networks, which means that hackers can find out the user's details. Block Chain protects addresses in a given wallet by constantly changing addresses which makes tracking payments or transactions difficult [20].

\section{F. Ease of Use}

Block Chain has the benefit of being easy to use and fast with competent integration capabilities. Data or money flow is faster due to the lack of intermediaries. In conventional banks, large-volume transactions take several days to complete, due to various protocols or software transfer. In addition to that, financial institutions have established working hours while in some countries on-line transactions are still not permitted on holidays. Block Chain technology works on a '247' based basis, meaning transactions can be made quickly and safely any day or any time.

\section{G. Transparency}

Users can verify and track public, decentralized ledger transactions. Any person can access all transaction records. Besides, any data modification is extremely difficult as the program is open source. Any modification in the logged data is sure to be seen with multiple eyes glued to the network which adds to the transparency and protection of Block Chain [21].

\section{DISADVANTAGES OF BLOCKCHAIN}

Block Chain technology has changed a variety of industries and aims to shape the future of Automation, Robotics, Machine learning and many other fields. But in the world of block chains this isn't all bliss and harmony. It has its limitations just like any other technology. We listed few drawbacks of Block Chain [22].

a. Slower process: Block chain can slow down when there are too many users on the network

b. High energy Consumption: Some solutions consume too much energy

c. Inefficient: Block chains are sometimes inefficient due to how they operate

d. High cost: Block chain implementation is a costly process

e. Interoperability: Block chain doesn't offer interoperability as of now f. Harder to Scale: Block chains are harder to scale due to their consensus method

g. Data is immutable: Block chain cannot go back as data is immutable

h. Self-maintenance: Users have to maintain their own wallets or else they can lose access

i. Still not mature: It still has a long way to go before it matures and get standardized

j. Integration: It is hard to integrate into legacy systems

\section{BITCOIN VS BLOCKCHAIN}

Bit Coin is the digital currency that uses crypto currencies and is regulated by the decentralized authority and is not like the currencies issued by the government, whereas the Block Chain is the form of ledger and tracks all the transactions taking place and helps promote Peer to Peer transactions. Bit Coin is a crypto currency and can be called a crypto-currency as well. It was developed primarily to speed up cross-border transactions, reduce the influence of the government over the transaction, and simplify the entire process without requiring third party intermediaries [10].

Bit Coin is also not a formally accepted means of payment but it is used by people all over the world for various types of transactions. It's very safe and secure because it's not physically present, and Block Chain is the best way to do those transactions. The Block Chain is a form of ledger that records all transactions and assists in Peer to Peer transactions. It is free, safe and available to all.

Block Chain thus serves as the registry of Bit Coin and accounts for all Bit Coin transactions. Block Chain has since evolved from strength to strength and is now catering for even small transactions across various industries. Block Chain has made it possible to adapt the technology to different areas and industries, so that the technology can be changed according to the high quality standard required by each company [15].

\section{CONCLUSION}

For its decentralized architecture and Peer to Peer existence, the block chain is highly regarded and endorsed. Nonetheless, Bit Coin covers other work on the block chain. But block chain may be extended far beyond Bit Coin to a number of fields. Block chain has demonstrated its ability to disrupt the conventional industry with its main characteristics: decentralization, sustainability, transparency.

Within this article, we're presenting a comprehensive block chain survey. First, we give an overview of the block chain technology like block chain architecture and the block chain key characteristics. Then we discuss the standard consensus algorithms used in the block chain. In different respects we evaluate and compare these protocols. We are also exploring applications common for block chains [20]. 


\section{International Journal of Engineering Applied Sciences and Technology, 2020 \\ Vol. 5, Issue 2, ISSN No. 2455-2143, Pages 543-548 \\ Published Online June 2020 in IJEAST (http://www.ijeast.com)}

Then, we list some of the obstacles and issues that would impede the growth of Block chain and summarize some of the current approaches to solving these problems. It also discusses some possible future directions. Smart contracts are fast evolving nowadays and many smart contract implementations are being proposed. Since there are many defects and weaknesses in smart contract languages, however, there are also groundbreaking applications Am nominated. However, because there are many defects and weaknesses in smart contract languages, it is hard to introduce many groundbreaking applications at the moment. We intend to conduct an in-depth investigation into the future of Smart contracts [21].

\section{REFERENCE}

[1] Zheng, Z., Xie, S., Dai, H. N., Chen, X., \& Wang, H. (2018). Blockchain challenges and opportunities: A survey. International Journal of Web and Grid Services, 14(4), 352-375.

[2] McGhin, T., Choo, K. K. R., Liu, C. Z., \& He, D. (2019). Blockchain in healthcare applications: Research challenges and opportunities. Journal of Network and Computer Applications.

[3] Crosby, M., Pattanayak, P., Verma, S., \& Kalyanaraman, V. (2016). Blockchain technology: Beyond bitcoin. Applied Innovation, 2(6-10), 71.

[4] Zheng, Z., Xie, S., Dai, H., Chen, X., \& Wang, H. (2017, June). An overview of blockchain technology: Architecture, consensus, and future trends. In 2017 IEEE international congress on big data (BigData congress) (pp. 557-564). IEEE.

[5] Pilkington, M. (2016). Blockchain technology: principles and applications. In Research handbook on digital transformations. Edward Elgar Publishing.

[6] Tian, F. (2016, June). An agri-food supply chain traceability system for China based on RFID \& blockchain technology. In 2016 13th international conference on service systems and service management (ICSSSM) (pp. 1-6). IEEE.

[7] Lemieux, V. L. (2016). Trusting records: is Blockchain technology the answer?. Records Management Journal.

[8] Wright, A., \& De Filippi, P. (2015). Decentralized blockchain technology and the rise of lex cryptographia. Available at SSRN 2580664.

[9] Biswas K, Muthukkumarasamy V. Securing smart cities using blockchain technology. In2016 IEEE 18th international conference on high performance computing and communications; IEEE 14th international conference on smart city; IEEE 2nd international conference on data science and systems (HPCC/SmartCity/DSS) 2016 Dec 12 (pp. 1392-1393). IEEE.
[10] Zheng, Z., Xie, S., Dai, H. N., Chen, X., \& Wang, H. (2018). Blockchain challenges and opportunities: A survey. International Journal of Web and Grid Services, 14(4), 352-375.

[11] Saberi, S., Kouhizadeh, M., Sarkis, J., \& Shen, L. (2019). Blockchain technology and its relationships to sustainable supply chain management. International Journal of Production Research, 57(7), 2117-2135.

[12] Sikorski, J. J., Haughton, J., \& Kraft, M. (2017). Blockchain technology in the chemical industry: Machine-to-machine electricity market. Applied Energy, 195, 234-246.

[13] Yaga, D., Mell, P., Roby, N., \& Scarfone, K. (2019). Blockchain technology overview. arXiv preprint arXiv:1906.11078.

[14] Ahram, T., Sargolzaei, A., Sargolzaei, S., Daniels, J., \& Amaba, B. (2017, June). Blockchain technology innovations. In 2017 IEEE Technology \& Engineering Management Conference (TEMSCON) (pp. 137-141). IEEE.

[15] Atzori, M. (2015). Blockchain technology and decentralized governance: Is the state still necessary?. Available at SSRN 2709713.

[16] Raval, S. (2016). Decentralized applications: harnessing Bitcoin's blockchain technology. " O'Reilly Media, Inc.".

[17] Zhang, Y., \& Wen, J. (2017). The IoT electric business model: Using blockchain technology for the internet of things. Peer-to-Peer Networking and Applications, 10(4), 983-994.

[18] Smith, A. To Analyse How Blockchain Technology can be used to Securely Store Different Information Sources.

[19] Nofer, M., Gomber, P., Hinz, O., \& Schiereck, D. (2017). Blockchain. Business \& Information Systems Engineering, 59(3), 183-187.

[20] Meng, W., Tischhauser, E. W., Wang, Q., Wang, Y., \& Han, J. (2018). When intrusion detection meets blockchain technology: a review. Ieee Access, 6, 1017910188.

[21] Risius, M., \& Spohrer, K. (2017). A blockchain research framework. Business \& Information Systems Engineering, 59(6), 385-409

[22] Yeoh, P. (2017). Regulatory issues in blockchain technology. Journal of Financial Regulation and Compliance. 